\title{
Formation of Star-Like and Core-Shell AuAg Nanoparticles during Two- and Three-Step Preparation in Batch and in Microfluidic Systems
}

\author{
J. Michael Köhler, ${ }^{1,2}$ H. Romanus, ${ }^{2}$ U. Hübner, ${ }^{3}$ and J. Wagner ${ }^{1}$ \\ ${ }^{1}$ Institute of Physics, Technical University Ilmenau, PF 100565, 98684 Ilmenau, Germany \\ ${ }^{2}$ Institute of Micro- and Nanotechnologies, Technical University Ilmenau, PF 100565, 98684 Ilmenau, Germany \\ ${ }^{3}$ Institute for Physical High Technology, 07745 Jena, Germany
}

Received 22 December 2006; Accepted 11 April 2007

Recommended by Berger Shlomo

Regular dendrit-like metal nanoparticles and core-shell nanoparticles were formed by the reduction of mixtures of tetrachloroaurate and silver nitrate solutions with ascorbic acid at room temperature in two- and three-step procedures. The formation of these particles was found in batch experiments as well as in micro flow-through processes using static micromixers. The characteristic diameters of 4-branched star particles were in the range between 60 and $100 \mathrm{~nm}$. The typical particles consist of four metal cores which are embedded in a common shell. Additionally, particles with five and more metallic cores were formed, to some extent, and aggregates of the 4-branched particles also were formed. Larger aggregates and network-like structures of connected star particles were formed after sedimentation. The properties of the formed particles are dependent on the educt concentrations as well as on the order of mixing steps and on the time interval between them. Obviously, the relation of nucleation and particle growth in relation to the concentrations of metal ions determines the composition and the properties of formed nanoparticles. So, star-like particles are observed in case of nucleation of $\mathrm{Au}$ in absence of silver ions but with silver deposition after short nucleation time. Spherical core shell particles are formed in case of silver salt addition after complete reduction of tetrachloroaurate in flow-through experiments with sufficient residence time between both mixing steps. Polymer layers are always found in the form of a second outer shell even if the polymer solutions are added in an early stage of particle formation.

Copyright (c) 2007 J. Michael Köhler et al. This is an open access article distributed under the Creative Commons Attribution License, which permits unrestricted use, distribution, and reproduction in any medium, provided the original work is properly cited.

\section{INTRODUCTION}

Metal nanoparticles are of interest due to their unique optical and chemical properties. They are of particular importance for molecular nanotechnology and for the labelling of biochips [1-4]. The properties of nanoparticles depend on composition $[5,6]$, size, as well as on the shape of the particles. Particularly, the resonant interaction with photons is strongly affected by geometrical parameters. This results in shape-dependent [7], orientation-dependent, and incidence direction-dependent light scattering, absorption, and colour of nanoparticles. In case of core-shell particles, the optical properties can be controlled by choosing the ratio between core diameter and shell thickness. Investigations with varied shell thickness show that the maximum of electromagnetic absorption can be controlled by tuning the thickness of metallic shells of spheric core-shell nanoparticles. The optical properties of nanoparticles are dependent generally on their different geometrical parameters and the chemical composition [8].

The shape of metal nanoparticles is of interest for nanotechnological applications (isotropy or anisotropy of opti$\mathrm{cal}$ and electronic properties), for the aggregation and selforganization behavior as well as for their catalytic activity. Nonspherical gold/silver nanoparticles were prepared under different reaction conditions. So, triangular and polyhedral nanoparticles were obtained in the presence of leaf extracts [9]. A large spectrum of new metal nanoparticles and of nanoparticles with special molecular shells was developed by using reaction mixtures containing thioles and surfactants $[10,11]$.

The quality of nanoparticles generated in solution processes is strongly affected by the local chemical conditions for nucleation and core growth. Therefore, the local 
concentrations of metal ions, ligands, and reducing agent are of great importance. In many cases, the nucleation is transport-controlled and occurs very quickly if the mixing is efficient. Thus, the local concentration gradients during the mixing of educt solutions are mainly responsible for the composition and size distribution of cores. Therefore, here, the formation of binary composed nanoparticles ( $\mathrm{Au} / \mathrm{Ag}$ ) was studied in dependence on mixing educts solution under different mixing conditions.

\section{EXPERIMENT}

The formation of binary composed metal nanoparticles was studied by batch and micro flow-through experiments. The addition of gold- and silver-containing solutions to the reducing agent occurred not simultaneously but successively in order to study the effect of mixing order. The application of microreactors is of particular interest for timecritical two-step processes. It was shown that micro flowthrough arrangements can be applied for the formation of gold nanoparticles [12, 13], silver nanoparticles [14], and for semiconductor nanoparticles [15]. Here, the deposition of a silver shell around a spherical gold core can be achieved simply in a flow-through arrangement with a residence channel between the chip mixer, in which the tetrachloroaurate is mixed with the ascorbic acid, and silver nitrate.

Due to the different behavior of both metals, inhomogeneous composed particles must be expected in case of mixed educt solutions. Beside the composition of educt solutions, the mixing procedure should have an influence on the product properties [16]. This expectation was confirmed by the experiments. The spectral properties of product solutions and the composition and shape of nanoparticles depend not only on the composition and on concentration ratios of educt solutions but also on the speed and order of educt mixing.

\section{MATERIAL AND METHODS}

All applied chemicals were p.A. graded. We used freshly prepared aqueous solutions. Reactions were carried out either in Eppendorf cups or in a microfluidic arrangement. The mixing in the cups was promoted by shaking or by using of a lab vortexer.

A microreaction system was used for the preparation of core-shell nanoparticles. It consisted of four syringe pumps, a two-step static micro mixer, a residence loop, and a $\mathrm{T}$ connector (Figure 1). For mixing, a chip-based static micro mixer (IPHT Jena, Germany, [17]) constructed in a glass/Si/glass sandwich configuration [18] was applied. The static micro mixer is based on the split-and-recombine principle. It was prepared in a sandwich chip technique including two glass chips and a silicon chip. The microchannels were etched by microlithographic techniques. The width of glass channels structures (half-circle-like cross-sections) amounts to $0.5 \mathrm{~mm}$ for the larger channel sections and $0.3 \mathrm{~mm}$ for the smaller channel sections. The sizes of the Si channels (rectangular cross-sections) are 0.2 and $0.3 \mathrm{~mm}$. The total

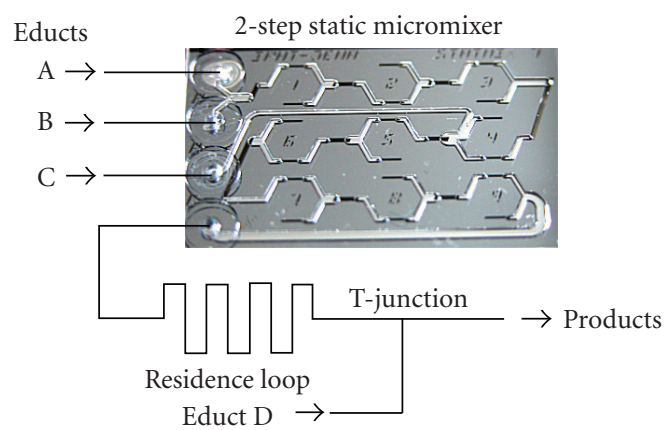

Figure 1: Experimental arrangement for the micro flow-through synthesis of core-shell nanoparticles $(A, B, C, D, \ldots$ inlet ports for educt solutions), static micromixer developed by J. M. Köhler and J. Albert.

channel length of one split-and-recombine unit amounts to about $10 \mathrm{~mm}$. The used device is equipped with two mixing zones. The first and the second educt solutions are mixed by four split and recombine zones before the third educt solution is added. After the input of the third educt, the whole reaction liquid passes through another five splitand-recombine zones. In the flow-through experiments, a PVA solution $(0.5 \%)$ was conducted to the first fluid inlet port (Figure $1,(\mathrm{~A}))$, a solution of ascorbic acid $(0.1 \mathrm{~mol} / \mathrm{L})$ was applied at the second port (B), and $\mathrm{HAuCl}_{4}$ solution $(0.5 \mathrm{mmol} / \mathrm{L})$ was added through the third port $(\mathrm{C})$. After completion of mixing, the reaction mixture was conducted through a residence loop (about $30 \mathrm{~cm}$ length, inner diameter is $0.5 \mathrm{~mm}$ ) before a $\mathrm{AgNO}_{3}$ solution $(1 \mathrm{mM})$ was added by the $\mathrm{T}$-junction (inner diameter $0.5 \mathrm{~mm}$ ). The residence loop between the outlet of the mixer chip and the T-junction at which the silver salt solution is added was long enough that the gold ions are reduced completely to gold particles inside the streaming liquid before the silver ions were introduced into the fluid system. The excess of reducing agent (ascorbic acid) ensures that the gold complex as well as the silver ions are reduced completely to the metal state. Fast pipetting in Eppendorf cups, shaking, or vortexing were used for mixing in batch experiments. All experiments were executed at room temperature.

The obtained colloidal solutions were characterized by spectrophotometry (Specord/Analytik Jena, 07745 Jena, Germany). Single particles, particle aggregates, and selected particle populations were characterized by scanning electron microscopy (Jeol Ltd., Tokyo 196-8558, Japan) and by transmission electron microscopy (Philips TECNAI, Eindhoven, The Netherlands).

A complex reaction behavior in dependence on the ratio of both metals was found if gold and silver salt solutions were premixed before bringing them together with the reducing agent. For this experiment series, an aqueous tetrachloroaurate solution $(0.5 \mathrm{mmol} / \mathrm{L})$ and an aqueous silver nitrate solution $(1 \mathrm{mmol} / \mathrm{L})$ were mixed in different ratios. Therefore, metal salt solutions with molar ratios of $\mathrm{Au} / \mathrm{Ag}$ between 0.25 and 2 were prepared. 


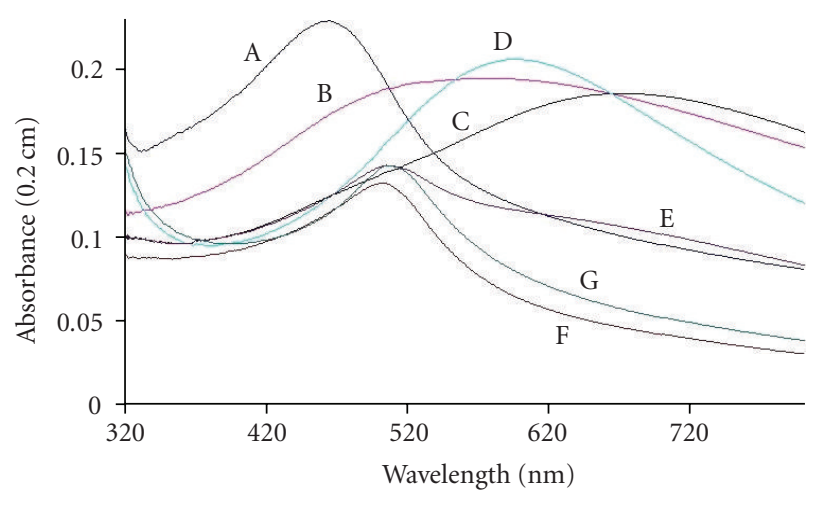

FIGURE 2: Optical absorption spectra of colloidal solutions obtained by reduction of different mixtures of $\mathrm{HAuCl}_{4}$ and $\mathrm{AgNO}_{3}$ solutions with ascorbic acid at room temperature: (A) $0.2 \mathrm{mM} \mathrm{Au} / 0.8 \mathrm{mM}$ Ag, (B) $0.25 \mathrm{mM} \mathrm{Au} / 0.5 \mathrm{mM} \mathrm{Ag}$, (C) $0.275 \mathrm{mM} \mathrm{Au} / 0.45 \mathrm{mM} \mathrm{Ag}$, (D) $0.3 \mathrm{mM} \mathrm{Au} / 0.4 \mathrm{mM} \mathrm{Ag}$, (E) $0.325 \mathrm{mM} \mathrm{Au} / 0.35 \mathrm{mM} \mathrm{Ag}$, (F) $0.35 \mathrm{mM} \mathrm{Au} / 0.3 \mathrm{mM} \mathrm{Ag}$, (G) $0.4 \mathrm{mM} \mathrm{Au} / 0.2 \mathrm{mM} \mathrm{Ag}$.

\section{RESULTS AND DISCUSSION}

Different product characteristics were observed when these metal salt solutions were reacted in a volume ratio of $1: 1$ with an ascorbic acid solution of an original concentration of $100 \mathrm{mmol} / \mathrm{L}$. At an $\mathrm{Au} / \mathrm{Ag}$ ratio of 0.25 , the optical spectrum of the product solution is dominated by an absorption peak with a maximum at about $460 \mathrm{~nm}$ (Figure 2, A). The position and shape of this absorption band suggest that it is due to nanoparticles, which are composed of a nearly homogeneous composition of gold and silver atoms. But, the influence of silver is dominating. This interpretation is supported by the maximum wavelength which was found between the typical absorption peak for pure Ag particles (around $420 \mathrm{~nm}$ ) and the peak for the plasmon band absorption of gold (about $525 \mathrm{~nm}$ ), but closer to the value of silver nanoparticles.

At a ratio of $\mathrm{Au} / \mathrm{Ag}$ of 0.9 or higher, the absorption of the product solution is characterized by an absorption at about $500 \mathrm{~nm}$ (Figure 2, spectra E, G, and F). Obviously, this indicates the increased influence of gold on the nanoparticle absorption in case of higher gold content. The peak presents a considerable high absorption in the short wavelength neighbourhood which is not only due to the silver content but also normally observed for pure Au nanoparticles. But, at an $\mathrm{Au} / \mathrm{Ag}$ ratio of a little less then one, a strong long wavelength shoulder arises in addition to the main absorption peak (Figure 2, E).

A dominating long wavelength peak was found if the molar ratio of $\mathrm{Au}$ to $\mathrm{Ag}$ is in the range of 0.6 and 0.8 (compare spectra $\mathrm{C}$ and $\mathrm{D}$ with the spectra $\mathrm{B}$ and $\mathrm{E}$ in Figure 2). In other word, the absorption spectrum first shifted bathochromically in case of increasing $\mathrm{Au} / \mathrm{Ag}$ ratio, but moved back to shorter wavelengths if the gold content is further increased. How can this behavior be understood? Long wavelength absorption of nanoparticles is mostly assigned to the formation of larger particles or aggregates, in which the electronic states of two or more particles are over-

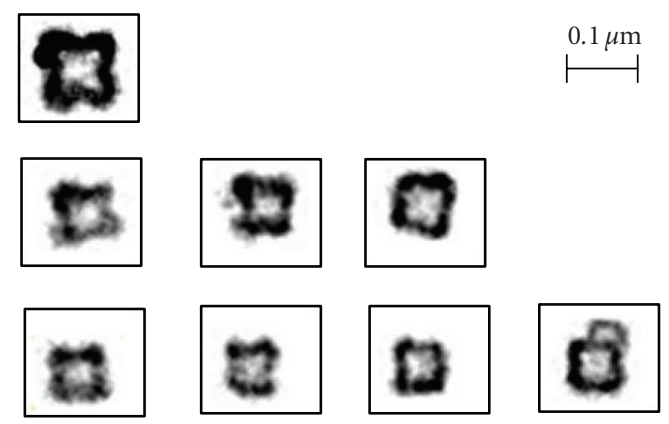

(a)

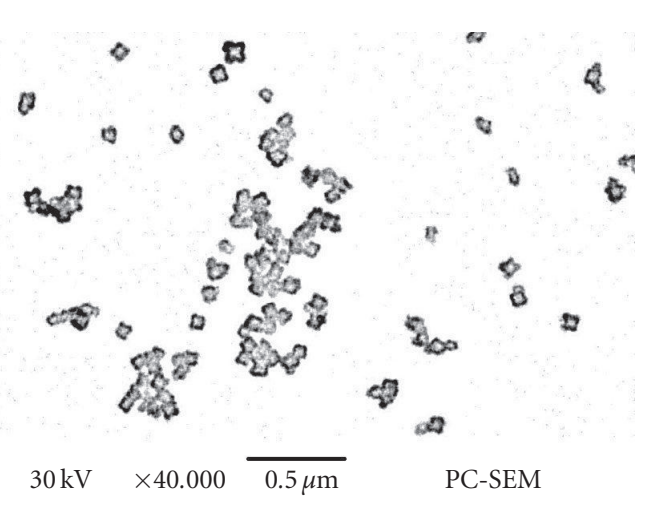

(b)

FIGURE 3: $\mathrm{Au} / \mathrm{Ag}$ nanoparticles from ascorbic acid reduction of mixed metal salt solution $\left(0.45 \mathrm{mmol} / \mathrm{L} \quad \mathrm{AgNO}_{3} / 0.275 \mathrm{mmol} / \mathrm{L}\right.$ $\mathrm{HAuCl}_{4}$ ) after adsorption on to a Si surface (inverted and contrastenhanced SEM images): (a) selected particles, (b) distribution of particles after adsorption into the Si surface.

lapping. Thus, it was assumed that the appearance of the broad long wavelength absorption indicates in our case the formation of larger particles or aggregates at mediate ratios of $\mathrm{Au} / \mathrm{Ag}$ too.

The assumption of formation of larger particles was confirmed by electron microscopic measurements of the products. Therefore, the colloidal solutions were centrifugated. The oily pellet was adsorbed onto the surface of polished monocrystalline silicon substrates and rinsed with water in order to remove all water-soluble components, namely residual ascorbic acid. At lower $\mathrm{Au} / \mathrm{Ag}$ ratios as well as at higher $\mathrm{Au} / \mathrm{Ag}$ ratios, single spherical particles and larger aggregates of them were found as known from similar investigations of the metal nanoparticle generation by ascorbic acid. But, in case of $\mathrm{Au} / \mathrm{Ag}$ ratios between 60 and 80\%, a considerable yield of typically featured larger nanoparticles was found (Figure 3(a)). The most often observed type was a star-like particle with four regular branches with a typical diameter between 80 and $120 \mathrm{~nm}$. The particles differ slightly in size, but only seldom in the number of corners. Figure 3(b) gives an impression of the size and shape distribution from a sample with high yield of size nanoparticles. The TEM images reflect a complex structure of the star-like particles (Figure 4). In all cases, the periphery of the particles shows a lower 


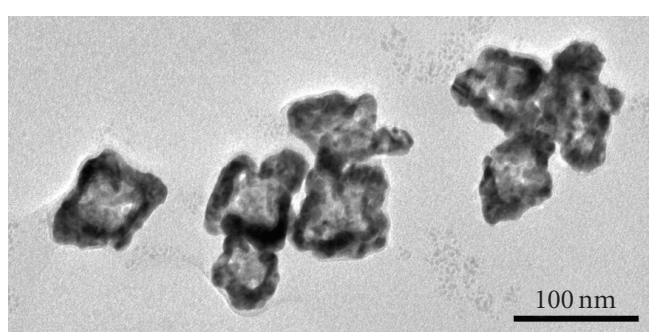

(a)
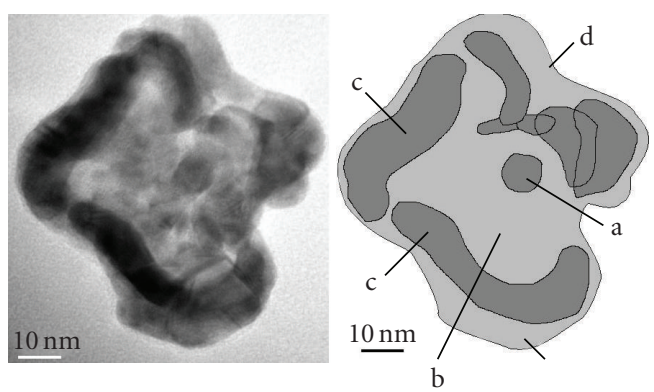

(b)

FIGURE 4: TEM image of star-like Au/Ag nanoparticles (same preparation conditions as in Figure 3): (a) ensembles of particles, (b) single particle at higher resolution (left original image, right: interpretation scheme for composition; a: spherical core with high density of heavy atoms (Au core), b: central part with lower concentration of heavy element (Ag-rich), c: peripheral parts with higher concentration of heavy atoms (Au-rich), d: outer shell, dominated by the less heavy metal $(\mathrm{Ag}))$.

electron transparency than the central part. This can be interpreted as an Au-rich zone in the outer part of the particles.

It is assumed that the nanostars - in contrast to larger aggregates - are not formed during the centrifugation, but are already present immediately after the particle formation due to the addition of ascorbic acid to the mixed metal salt solution. In some cases, particles with eight corners were found. They can be interpreted as dimers of the 4-branched star-like particles.

The complexity of the formation process of nanoparticles composed by gold and silver becomes clearer if we considered not only the educt composition but also the order and rate of the mixing of educt solutions. In contrast to the large long wavelength absorption which arose if $\mathrm{Au}$ and $\mathrm{Ag}$ are premixed in a concentration ratio of $1: 2$, completely other product spectra were found if tetrachloroaurat and ascorbic acid were mixed and silver nitrate was added later. At the same concentration ratio, a strongly silver-dominated sharp plasmon peak (at about $400 \mathrm{~nm}$ ) arose if the silver salt was added later to the reaction solution of gold salt and reducing agent, about 1 second after starting the gold reduction (spectra A and B in Figure 5). Only a slight shoulder at $500 \mathrm{~nm}$ is observed. This shoulder became more intensive if the time between adding the gold salt and the silver salt to the ascorbic acid solution is reduced below one second. At the same time, the silver-dominated absorption peak

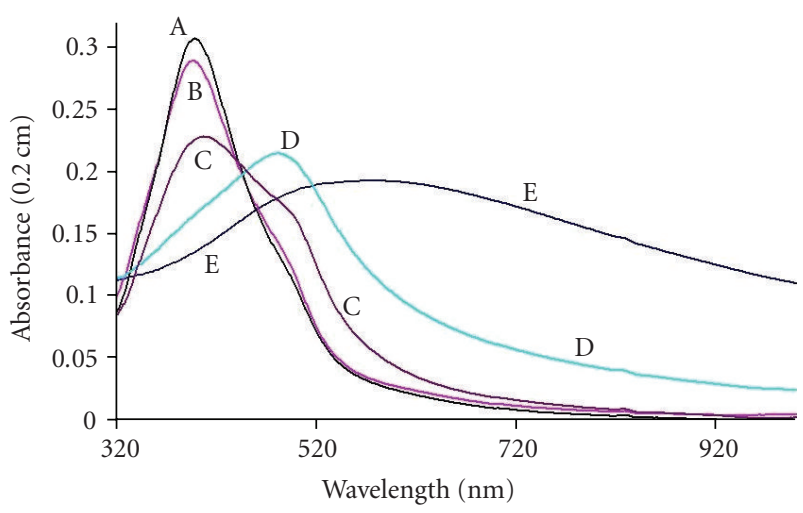

\begin{abstract}
A: premix $\mathrm{Au}+\mathrm{AA}, \mathrm{Ag}$ after $4 \mathrm{~s}$ B: premix $\mathrm{Au}+\mathrm{AA}, \mathrm{Ag}$ after $1 \mathrm{~s}$ C: premix $\mathrm{Au}+\mathrm{AA}, \mathrm{Ag}$ after $0.5 \mathrm{~s}$ D: premix Au + AA, Ag after $0.3 \mathrm{~s}$ E: premix $\mathrm{Au}+\mathrm{Ag}, \mathrm{Ag}$ after $60 \mathrm{~s}$
\end{abstract}

FIGURE 5: Influence of intermediate time and mixing order on the quality of product solutions reflected by absorption spectra (batch experiments, $\mathrm{Au}: \mathrm{HAuCl}_{4}$ solution, $\mathrm{AA}$ ascorbic acid solution, Ag: $\mathrm{AgNO}_{3}$ solution). $100 \mu \mathrm{l}$ each solution contains $0.5 \mathrm{mM} \mathrm{HAuCl}_{4}$, $1 \mathrm{mM} \mathrm{AgNO}_{3}$, and $0.1 \mathrm{M}$ ascorbic acid

is considerably reduced (spectrum $\mathrm{C}$ in Figure 5). A further reduction of intermediate time down to about 0.3 second (and without any change in the concentrations of educt solutions) leads to an inversion of the peak shoulder relation. The longer wavelength shoulder at about $500 \mathrm{~nm}$ became the dominating peak, whereas the short wavelength absorption at about $400 \mathrm{~nm}$ was decreased to a shoulder of the longer wave-length band (spectrum D in Figure 5).

The observations can only be interpreted by a strong kinetic effect on the quality of colloidal product solutions. This can be understood if we keep in mind that the reduction of silver ions by ascorbic acid at room temperature is strongly kinetically hindered, whereas tetrachloroaurate very quickly forms gold nanoparticles under analogous conditions. It is well known that in the presence of metallic gold and also in case of gold nanoparticles the rate of silver ion reduction can be enhanced dramatically (metal-catalyzed chemical reduction of silver ions is known from photographic development long time ago). This difference between the behavior of gold and silver leads to fast nucleation by reduction with ascorbic acid at room temperature in the presence of gold salt or gold nanoparticles, but no nucleation appens in the absence of gold. The observed differences in product quality and optical spectra can be discussed in the context of these behaviors. Pure gold particles nucleate and form the typical gold nanoparticles if tetrachloroaurate and the reducing agent are premixed first. If silver salt is added later, metallic silver is deposited at the gold cores. The optical properties of the obtained $\mathrm{Au} / \mathrm{Ag}$ nanoparticles are dominated by the silver-rich shell of the particles, whereas the gold core obviously possesses only small influence on the particle plasmon spectra (small and sharp short wavelength absorption peak 


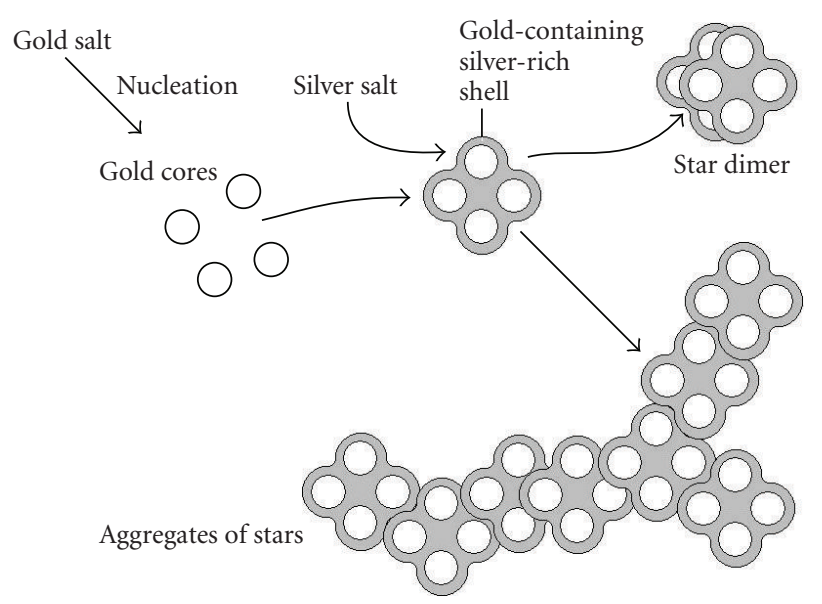

FIGURE 6: Proposal of a mechanism for the formation of 4-branched star-like particles and fractal particle aggregates in case of reduction of mixed gold and silver ion-containing solution.

in spectra A and B in Figure 5). Only if the Au nuclei do not have sufficient time to grow under complete consumption of tetracholoroaurate before the addition of silver salt, the outer part of the nanoparticles is composed by gold and silver atoms. Therefore, the spectra of the formed particles and their colloidal solution are characterized by a plasmon peak with a mixed composition characteristic (spectra $\mathrm{C}$ and $\mathrm{D}$ in Figure 5). A similar situation is found if tetrachloroaurate and silver nitrate are mixed before the reducing agent was added. The absorption peaks of the obtained colloidal solutions were broader as in the strongly silver-dominated particles and possessed always absorption maxima between the peaks of pure gold and pure silver particles (spectra A, F, $\mathrm{G}$ in Figure 2). Only at certain mediate concentration ratios, a special situation seems to arise, which causes the formation of the characteristic star-like particles. It is supposed that the formation of this particles has to occur with primary formation of gold-rich cores, which aggregate regularly in quartets, that contain a silver-rich center and are subsequently covered with a silver-rich thin shell (scheme in Figure 6). This interpretation is supported by the electron micrographs suggesting a higher silver content in the shell of the star-like particles and cores consisting mainly or exclusively of gold.

The formation of $\mathrm{Au} / \mathrm{Ag}$ nanoparticles under condition of sufficient time for gold reduction was studied by batch experiments with addition of $\mathrm{AgNO}_{3}$ after completion of $\mathrm{Au}$ reduction (delay of some minutes between $\mathrm{Au}$ nucleation and silver nitrate addition) and in separate experiments by the use of the microfluidic arrangement (low flow rates). In this case, no star-like particles but spherical core-shell particles were obtained. Obviously, the addition of water-soluble polymers supports the formation of rather regular spherical particles. The mixing rate of static micro mixer is strongly dependent on the flow rate. The mixing time in one mixing unit can be lowered from about 1 second (for flow rates of about $100 \mu \mathrm{L} /$ minute) down to less than 50 milliseconds (for flow rates above $2 \mathrm{~mL} / \mathrm{minute}$ ).

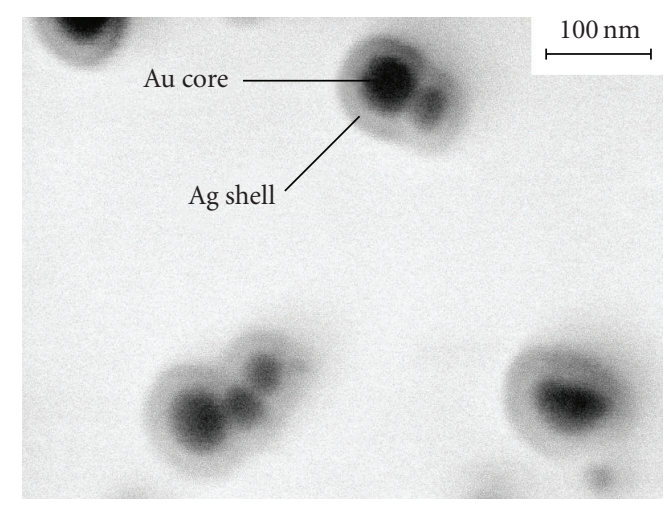

(a)

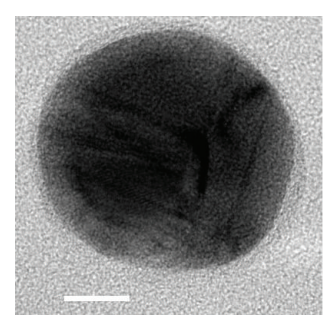

$10 \mathrm{~nm}$

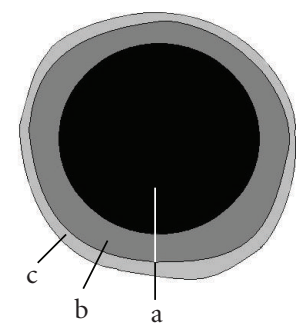

(b)
Figure 7: Core-shell metal nanoparticles obtained by application of the experimental arrangement of Figure 1; (a) inverted lowvoltage SEM image (flow rates: A (PVA), B (ascorbic acid), C (tetrachloroauric acid), D (silver nitrate): $50 \mu \mathrm{L} /$ minute), (b) TEM image of a single particle and scheme of interpretation (flow rates: $\mathrm{A}$ (PVA): $20 \mu \mathrm{L} /$ minute, $\mathrm{B}$ (ascorbic acid), C (tetrachloroauric acid), $\mathrm{D}$ (silver nitrate): $50 \mu \mathrm{L} / \mathrm{min}$ ), a denotes heavy metal core $(\mathrm{Au}), \mathrm{b}$ denotes inner shell (Ag-rich), c denotes outer shell: polymer.

The obtained nanoparticles were characterized by lowvoltage SEM and TEM in order to distinguish different metal materials due to their material contrast. The inverted low-voltage SEM images show particles with a dark core and a brighter grey shell (Figure $7(\mathrm{a})$ ). This observation corresponds well with the suggestion of $\mathrm{Au} / \mathrm{Ag}$ core-shell nanoparticles formed during the continuous flow-through synthesis. This interpretation is supported by TEM images (Figure $7(\mathrm{~b})$ ). They suggest a higher concentration of the heavier metal in the center (Au core) and a higher concentration of the less heavy metal in the shell. In addition, TEM images of particles generated at the presence of PVA show a second outer shell with low electron absorption. The low contrast of this outer shell speaks for the predominance of light elements, which can be well discussed as the adsorbed hydrocarbon macromolecules. The thickness of this layer was found in the range of $2.5-4 \mathrm{~nm}$ (Figure 8).

The reduction of tetrachloroauric acid and silver nitrate with ascorbic acid at room temperature represents a special case of formation of binary composed nanoparticles. The nucleation rate of silver is very low under the applied 


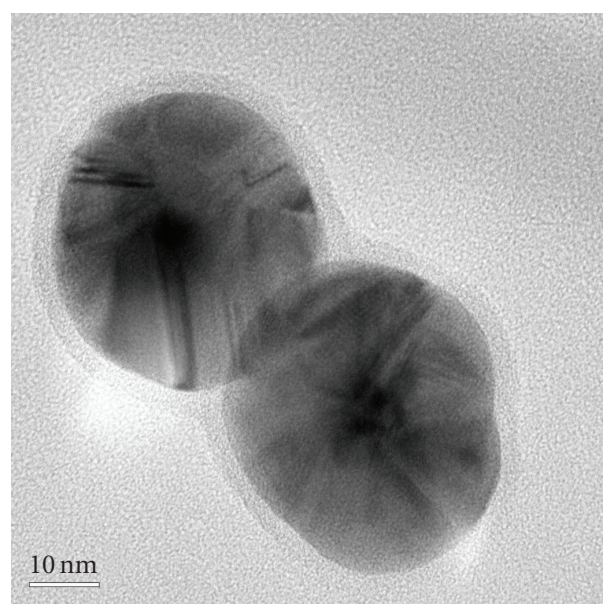

(a)

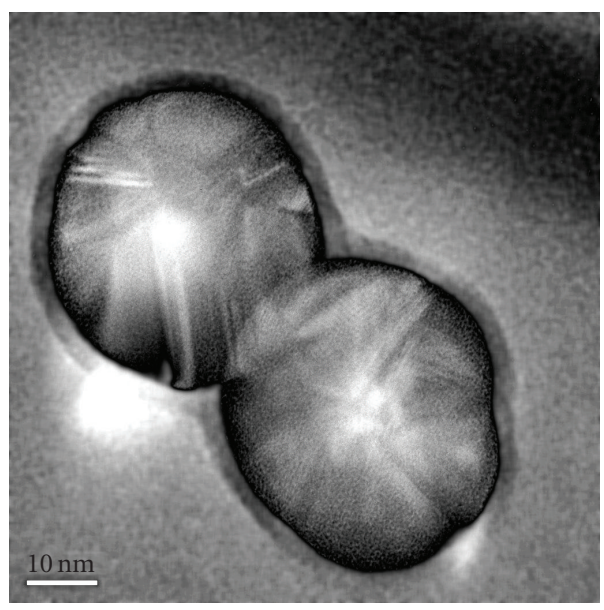

(b)

FIGURE 8: TEM image of a twin particle (flow-through preparation, conditions as in Figure 7(b)): (a) original image, (b) after image processing.

conditions (ascorbic acid concentration up to $0.1 \mathrm{~mol} / \mathrm{L}$ ). In contrast, gold cores are quickly formed under the same conditions. But in case of a sufficient concentration of reducing agent, silver ions were comparatively fast reduced, if gold cores are present. This effect is due to the catalytic effect of metal cores on the silver ion reduction, which is kinetically hampered in homogeneous phase. This behavior should cause a primary formation of gold cores in all cases of mixed metal salt educt solutions. After this nucleation phase, growth processes can take place incorporating simultaneously silver and gold species present in the solution. The composition of shells depends on the remaining metal complex concentrations in the solution. As a result, spatial distribution of elements inside the nanoparticles must be regarded as a product of nucleation, particle growth, and particle aggregation under the specific conditions of educt mixing.

\section{ACKNOWLEDGMENTS}

The authors would like to thank the German Environment Foundation (DBU) for the financial support. The stimulating discussions with W. Fritzsche and G. A. Gross as well as the technical support by G. Mayer, J. Albert, and F. Möller are gratefully acknowledged.

\section{REFERENCES}

[1] T. A. Taton, C. A. Mirkin, and R. L. Letsinger, "Scanometric DNA array detection with nanoparticle probes," Science, vol. 289, no. 5485, pp. 1757-1760, 2000.

[2] R. Möller, A. Csáki, J. M. Köhler, and W. Fritzsche, "DNA probes on chip surfaces studied by scanning force microscopy using specific binding of colloidal gold," Nucleic Acids Research, vol. 28, no. 20, p. e91, 2000.

[3] C. M. Niemeyer and D. Blohm, "DNA-Mikroarrays," Angewandte Chemie, vol. 111, no. 19, pp. 3039-3043, 1999.

[4] W. Fritzsche, "DNA-gold conjugates for the detection of specific molecular interactions," Reviews in Molecular Biotechnology, vol. 82, no. 1, pp. 37-46, 2001.

[5] S. Link, Z. L. Wang, and M. A. El-Sayed, "Alloy formation of gold-silver nanoparticles and the dependence of the plasmon absorption on their composition," Journal of Physical Chemistry B, vol. 103, no. 18, pp. 3529-3533, 1999.

[6] C. Sönnichsen and A. P. Alivisatos, "Gold nanorods as novel nonbleaching plasmon-based orientation sensors for polarized single-particle microscopy," Nano Letters, vol. 5, no. 2, pp. 301-304, 2005.

[7] S. Link and M. A. El-Sayed, "Spectral properties and relaxation dynamics of surface plasmon electronic oscillations in gold and silver nanodots and nanorods," Journal of Physical Chemistry B, vol. 103, no. 40, pp. 8410-8426, 1999.

[8] G. Schmid and L. F. Chi, "Metal clusters and colloids," Advanced Materials, vol. 10, no. 7, pp. 515-526, 1998.

[9] M. Moskovits, I. Srnová-Šloufová, and B. Vlčková, "Bimetallic Ag-Au nanoparticles: extracting meaningful optical constants from the surface-plasmon extinction spectrum," Journal of Chemical Physics, vol. 116, no. 23, pp. 10435-10446, 2002.

[10] M. Brust and C. J. Kiely, "Some recent advances in nanostructure preparation from gold and silver particles: a short topical review," Colloids and Surfaces A, vol. 202, no. 2-3, pp. 175-186, 2002.

[11] P. Moriarty, M. D. R. Taylor, and M. Brust, "Nanostructured cellular networks," Physical Review Letters, vol. 89, no. 24, Article ID 248303, 4 pages, 2002.

[12] J. Wagner, T. Kirner, G. Mayer, J. Albert, and J. M. Köhler, "Generation of metal nanoparticles in a microchannel reactor," Chemical Engineering Journal, vol. 101, no. 1-3, pp. 251260, 2004.

[13] J. Wagner and J. M. Köhler, "Continuous synthesis of gold nanoparticles in a microreactor," Nano Letters, vol. 5, no. 4, pp. 685-691, 2005.

[14] X. Z. Lin, A. D. Terepka, and H. Yang, "Synthesis of silver nanoparticles in a continuous flow tubular microreactor," Nano Letters, vol. 4, no. 11, pp. 2227-2232, 2004.

[15] H. Nakamura, Y. Yamaguchi, M. Miyazaki, M. Uehara, H. Maeda, and P. Mulvaney, "Continuous preparation of CdSe nanocrystals by a microreactor," Chemistry Letters, vol. 31, no. 10, pp. 1072-1073, 2002. 
[16] J. M. Köhler, M. Held, U. Hübner, and J. Wagner, "Formation of Au/Ag nanoparticles in a two step micro flow-through process," Chemical Engineering \& Technology., vol. 30, no. 3, pp. 347-354, 2007.

[17] N. Schwesinger, T. Frank, and H. Wurmus, "A modular microfluid system with an integrated micromixer," Journal of Micromechanics and Microengineering, vol. 6, no. 1, pp. 99-102, 1996.

[18] T. Kirner, J. Albert, M. Günther, G. Mayer, K. Reinhäckel, and J. M. Köhler, "Static micromixers for modular chip reactor arrangements in two-step reactions and photochemical activated processes," Chemical Engineering Journal, vol. 101, no. 1-3, pp. 65-74, 2004. 

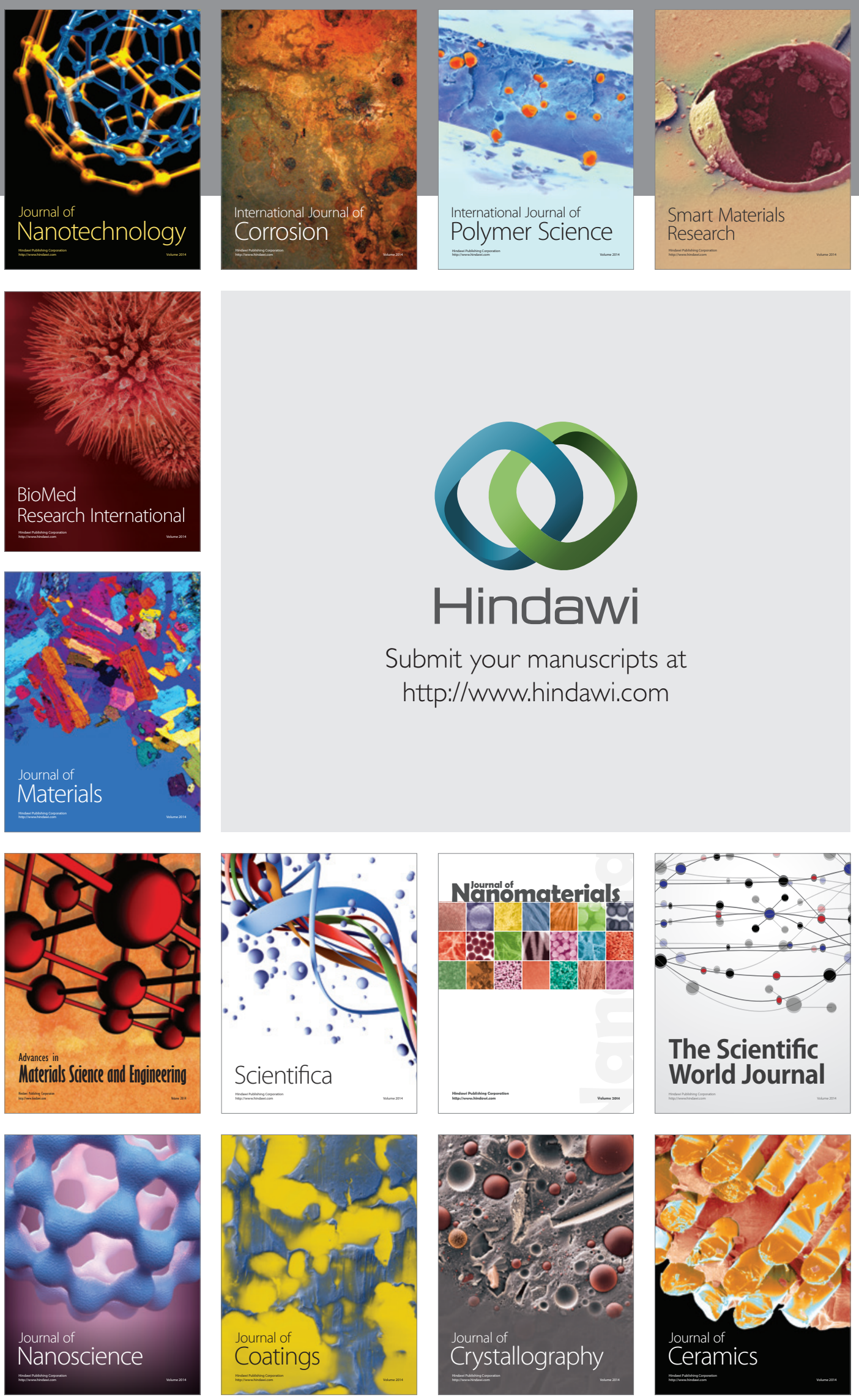

The Scientific World Journal

Submit your manuscripts at

http://www.hindawi.com

\section{World Journal}

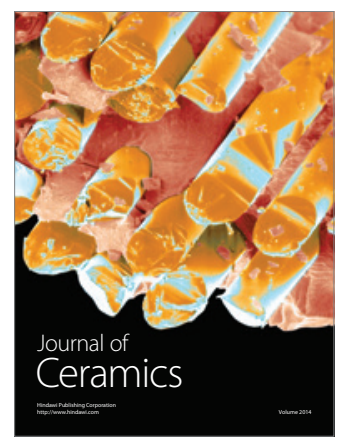

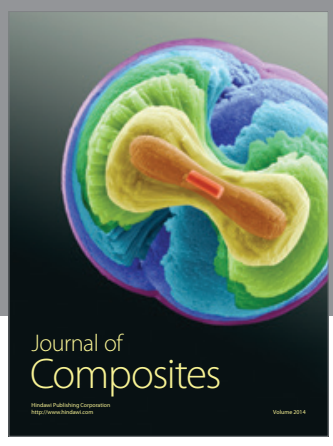
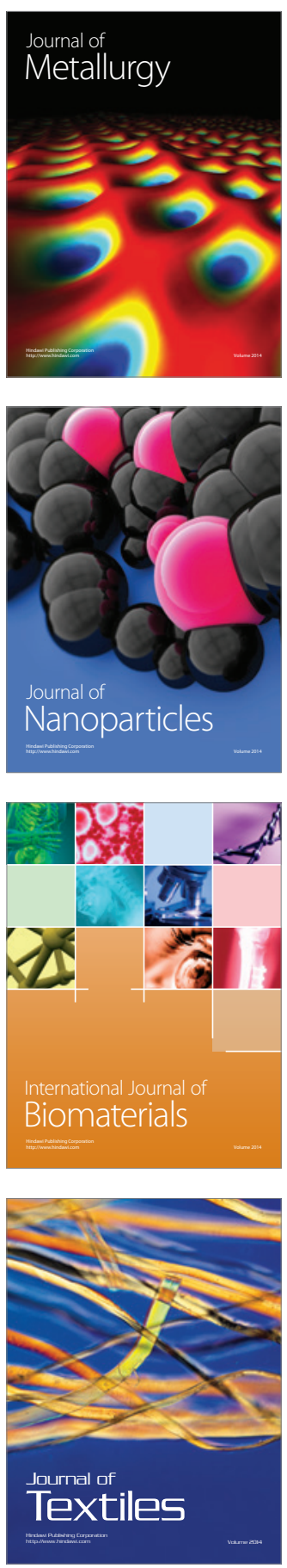